Proceedings of the

International Geometry Center

Vol. 11, no. 4 (2018) pp. 1-17

\title{
Nonpositive curvature foliations on 3-manifolds with bounded total absolute curvature of leaves
}

\author{
Dmitry V. Bolotov
}

\begin{abstract}
In this paper we introduce a new class of foliations on Riemannian 3-manifolds, called $B$-foliations, generalizing the class of foliations of nonnegative curvature. The leaves of $B$-foliations have bounded total absolute curvature in the induced Riemannian metric. We describe several topological and geometric properties of $B$-foliations and the structure of closed oriented 3 -dimensional manifolds admitting $B$-foliations with non-positive curvature of leaves.
\end{abstract}

Анотація. В даній роботі вводиться новий клас шарувань, узагальнюючий клас шарувань невід'ємної кривини, на 3-вимірних орієнтованих замкнутих ріманових многовидах, які ми називаємо $B$-шаруваннями. Це трансверсально орієнтовані шарування, шари яких мають обмежену абсолютну повну кривину шарів відносно ріманової метрики індукованої деякою рімановою метрикою заданою на всьому 3-многовиді. Зауважимо, що шарування невід'ємної кривини мають обмежену абсолютну повну кривину, тобто є $B$-шаруваннями, за відомою теоремою Кон-Фоссена. У роботі описано топологічні та геометричні властивості $B$-шарувань та показано, що їх структура дуже схожа на структуру шарувань невід'ємної кривини. Зокрема доведено, що $B$-шарування, як і шарування невід'ємної кривини, є шаруваннями майже без голономії. Також встановлено, що шар $B$-шарування, який є граничним для деякого іншого шара, як і у випадку шарувань невід'ємної кривини, повинен бути плоским. Звідси випливає, що щільне $B$-шарування є плоским.

Особливу увагу в роботі приділено $B$-шаруванням недодатної кривини. Такі шарування позначаються через $B_{\leq 0}$-шарування. Як основний результат в роботі доведено, що трансверсально орієнтоване $B_{\leq 0}$-шарування на орієнтованому замкнутому 3 -вимірному рімановому многовиді є або розшаруванням над колом з шаром гомеоморфним замкнутій поверхні $M^{2}$ недодатної кривини; або є щільним плоским шаруванням без голономії, а сам 3 -многовид $є$ торичним розшаруванням над колом;

2010 Mathematics Subject Classification: 57R30

Keywords: foliations, curvature of leaves, topology of 3-manifolds, Riemannian geometry DOI: http://dx.doi.org/ 
або існує скінченне число компактних шарів, гомеоморфних двовимірному тору, які розбивають многовид на частини гомеоморфні тотальному простору розшарування над колом з шаром, який є компактною поверхнею з межею недодатної ейлерової характеристики.

Також доводиться і зворотне твердження, яке говорить, що будь-яке орієнтоване розшарування над колом з шаром $M^{2}$, гомеоморфним орієнтованій поверхні недодатної ейлерової характеристики $є B_{\leq 0}$-шаруванням, та якщо орієнтований замкнутий 3 -многовид розбивається скінченним числом торів на частини, гомеоморфні тотальному простору розшарування над колом 3 шаром, який є компактною поверхнею $з$ межею недодатної ейлерової характеристики, то даний 3-многовид для деякої ріманової метрики має $B_{\leq 0}$-шарування, для якого дані тори будуть плоскими шарам.

Крім того, в роботі описано фундаментальну групу 3-многовиду, який має $B_{\leq 0}$-шарування та показано, що такі 3 -многовиди повинні бути асферичними i, на відміну від цього, доведено, що будь-який орієнтований 3многовид має трансверсально орієнтоване $B$-шарування.

\section{INTRODUCTION}

A foliation on a Riemannian manifold whose leaves have nonnegative (nonpositive) curvature in the induced metric will be called a foliation of nonnegative (nonpositive) curvature. Early we have proved the following theorem.

Theorem 1.1. [2] Let $M^{3}$ be a closed oriented Riemannian 3-manifold equipped with a codimension one transversely oriented $C^{\infty}$-foliation $\mathcal{F}$ of nonnegative curvature. Then $\mathcal{F}$ is a foliation almost without holonomy and $M^{3}$ is homeomorphic to one of the following manifolds:

1) toric bundle over the circle;

2) toric semibundle;

3) $S^{2} \times S^{1}$;

4) $\mathbb{R} P^{3} \# \mathbb{R} P^{3}$;

5) lens space $L_{p / q}$;

6) prismatic space.

Each of the listed spaces admits a nonnegative curvature foliation with respect to some Riemannian metric.

Remark 1.2. Recall that a toric semibundle is glued of two copies of oriented twisted $I$-bundles over the Klein bottle by the some diffeomorphism between their boundaries. Also a prismatic space is a spherical form which has a structure of a Seifert bundle over the orbifold $P(n)$, where $P(n)$ is a projective space with a single conical point of type $D^{2} / \mathbb{Z}_{n}$. 
Let $(L, g)$ be a complete noncompact surface endowed with a smooth Riemannian metric. Then the total curvature of $L$ is the improper integral $\int_{L} K d \mu$ of the Gaussian curvature $K$ with respect to the volume element $d \mu$ of $(L, g)$. One sais that $L$ admits a total curvature if for any compact exhaustion $\Omega_{i}$ of $L$, there exists finite or infinite limit

$$
\lim _{i \rightarrow+\infty} \int_{\Omega_{i}} K d \mu=\int_{L} K d \mu
$$

In [3] Cohn-Vossen also proved that

$$
\int_{L} K d \mu \leq 2 \pi \chi(L)
$$

provided that the Euler characteristic $\chi(L)$ of $L$ is finite.

In 1957 Huber established the following result.

Theorem 1.3. [9] Denote $K_{-}=\max \{-K, 0\}$. If

$$
\int_{L} K_{-} d \mu<\infty
$$

then $\int_{L} K d \mu$ exists and $L$ is homeomorphic to a compact Riemannian surface with finitely many punctures, so L has a finite topology.

From Huber's theorem follows, that the Cohn-Vossen inequality (1.2) holds for complete surfaces of nonnegative curvature, in particular for leaves of nonnegative curvature foliations. Observe that in this case the orientable leaves can be either spheres (if $\chi(L)=2$ ) or planes (if $\chi(L)=1$ ), or cylinders or tori (if $\chi(L)=0$ ).

It also follows from Huber's result that (1.3) is equivalent to the inequality $\int_{L}|K| d \mu<\infty$. The integral $\int_{L}|K| d \mu$ is called the total absolute curvature.

If 1964 Hartman proved the following Theorem.

Theorem 1.4. [7] Under assumption (1.3) the area growth of a geodesic ball of radius $r$ at a fixed point of $L$ is at most quadratic in $r$.

Also Li [13] shown that if $L$ has at most quadratic area growth, finite topology, and the Gaussian curvature of $L$ is either nonpositive or nonnegative near infinity of each end, then the total curvature of $L$ must be finite.

The purpose of this paper is to describe the structure of $C^{\infty}$-foliations $\mathcal{F}$ on a closed 3-manifold $M$ admiting a Riemannian metric in which the leaves of $\mathcal{F}$ have bounded total absolute curvature. Such foliations will be called $B$-foliations. We pay a special attention to nonpositive curvature 
$B$-foliations, which we call $B_{\leq 0}$-foliations. In this regard, let us recall the following result which is a foliated analogue of Cartan-Hadamard Theorem.

Theorem 1.5. [16] Let $\mathcal{F}$ be a codimension one $C^{3}$-foliation of nonpositive sectional curvature on a complete Riemannian $n$-manifold $M^{n}$. Then its universal covering $\widetilde{M}$ is diffeomorphic to $\mathbb{R}^{n}$.

As a consequence of this theorem one gets the following statement:

Theorem 1.6. [16] Let $\mathcal{F}$ be a codimension one $C^{3}$-foliation of nonpositive sectional curvature on a complete $n$-manifold $M^{n}$. Then for any leaf $L \in \mathcal{F}$ the homomorphism

$$
i_{*}: \pi_{1}(L) \rightarrow \pi_{1}\left(M^{n}\right)
$$

induced by the inclusion $i: L \subset M^{n}$ is a monomorphism. Hence the universal covering $\widetilde{\mathcal{F}}$ of the foliation $\mathcal{F}$ on the universal cover $\widetilde{M}$ consists of leaves diffeomorphic to $\mathbb{R}^{n-1}$.

In the present paper we prove the following theorem similar to Theorem 1.1 which describes the structure of $B_{\leq 0}$-foliations.

First recall that a graph of groups over a graph $Y$ is an assignment to each vertex $u$ of $Y$ a group $G_{u}$ and to each oriented edge $e=(u, v)$ of $Y$ a group $G_{e}$ together with two monomorphisms $\phi_{e, u}: G_{e} \rightarrow G_{u}$ and $\phi_{e, v}: G_{e} \rightarrow G_{v}$ mapping $G_{e}$ into the groups assigned to the vertices at its ends. If $T$ is a spanning tree for $Y$ (i.e a subree contained all of the vertices of $Y$ ), then the fundamental group of that graph of groups is the group generated by the vertex groups $G_{u}$ and elements $e$ for each edge of $Y$ with the following relations:

- $\bar{e}=e^{-1}$ if $\bar{e}$ is the edge $e$ with the reverse orientation.

- $e \phi_{e, u}(x) e^{-1}=\phi_{e, v}(x)$ for all $x \in G_{e}$.

- $e=1$ if $e \in T$.

This definition is independent of a choice of $T$.

Main Theorem. (1) Let $M$ be a closed orientable Riemannian 3-manifold and $\mathcal{F}$ be a transversely orientable smooth foliation being $B_{\leq 0}$ with respect to the Riemannian metric on $M$. Then the following statements hold true.

(a) $M$ is a $K(\pi, 1)$-space (that is $\pi_{1}(M)=\pi$ and $\pi_{i}(M)=0$ for $i \geq 2$ ).

(b) $\mathcal{F}$ is almost without holonomy.

(c) At least one of the following conditions is satisfied. 
(i) The leaves of $\mathcal{F}$ are fibers of some $M_{g}^{2}$-bundle over the circle, where $M_{g}^{2}$ is an oriented closed surface of genus $g \geq 1$;

(ii) $\mathcal{F}$ is flat ${ }^{1}$ and all its leaves are dense. In this case $M$ is homeomorphic to a total space of some torus bundle over the circle.

(iii) There exist finitely many compact leaves $\left\{K_{i}\right\}$ each homeomorphic to the torus $T^{2}$ such that every connected component $A_{j}$ of $\overline{M \backslash \cup_{i} K_{i}}$ is fibered over the circle with a fiber homeomorphic to some surface $S_{j}$ of nonpositive Euler characteristic with boundary. In this case every inclusion $K_{i} \subset A_{j}$ the induced homomorphism $\phi_{i, j}: \pi_{1} K_{i} \rightarrow \pi_{1} A_{j}$ is a monomorphism. Moreover, the fundamental group $\pi_{1}(M)$ is isomorphic to the fundamental group of the graph of groups $G$ whose vertices correspond to groups $\pi_{1}\left(A_{j}\right)$, the edges correspond to groups $\pi_{1}\left(K_{i}\right)$, and the "end" monomorphisms are $\phi_{i, j}$.

(2) Let $M$ be an oriented closed 3-manifold having one of the following properties:

(a) $M$ can be divided by a finite set of embedded tori into pieces $\left\{A_{j}\right\}$ each of which is fibered over the circle with a fiber homeomorphic to a compact surface $S_{j}$ with boundary and $\chi\left(S_{j}\right) \leq 0$;

(b) $M$ is a total space of an oriented $M_{g}^{2}$-bundle over the circle, where $M_{g}^{2}$ $i s$ an oriented closed surface of genus $g \geq 1$.

Then $M$ admits a $B_{\leq 0}$-foliation with respect to some Riemannian metric.

\section{Foliations}

2.1. Definition of foliation. Let $M$ be an $n$-dimensional manifold. Then a partition $\mathcal{F}=\left\{F_{\alpha}\right\}$ of $M$ into path-connected subsets (leaves) is called a foliation of dimension $p$ (or codimension $q$, where $p+q=n$ ) on $M$ if $\mathcal{F}$ admits an atlas $\mathcal{U}=\left\{\left(U_{\lambda}, \varphi_{\lambda}\right)\right\}_{\lambda \in \Lambda}$ consisting of foliated charts.

The latter means that each connected component of a leaf in a chart $\left(U_{\lambda}, \varphi_{\lambda}\right)$ with coordinates $\left(x_{1}, \ldots, x_{p}, y_{1}, \ldots, y_{q}\right)$ is given by

$$
y_{1}=\text { const, } \ldots, y_{q}=\text { const, }
$$

and the transition maps

$$
g_{i j}=\varphi_{i} \circ \varphi_{j}^{-1}: \varphi_{j}\left(U_{i} \cap U_{j}\right) \rightarrow \varphi_{i}\left(U_{i} \cap U_{j}\right)
$$

have the form

$$
g_{i j}(x, y)=\left(\hat{g}_{i j}(x, y), \bar{g}_{i j}(y)\right),
$$

where $x \in \mathbb{R}^{p}, y \in \mathbb{R}^{q}$, so the second coordinate function $\bar{g}_{i j}: \mathbb{R}^{p+q} \rightarrow \mathbb{R}^{q}$ does not depend on $y$.

\footnotetext{
${ }^{1}$ A "flatness" means that all leaves have zero Gaussian curvature in the induced Riemannian metric
} 
The atlas $\mathcal{U}=\left\{\left(U_{\lambda}, \varphi_{\lambda}\right)\right\}_{\lambda \in \Lambda}$ is supposed to be at least $C^{2}$-smooth and good in the sense that

1) $\mathcal{U}$ is locally finite;

2) $U_{\lambda}$ is relatively compact in $M$, and $\varphi_{\lambda}\left(U_{\lambda}\right)=(-1,1)^{n} \subset \mathbb{R}^{n}$;

3) $\overline{U_{i} \cup U_{j}} \subset W_{i j}$, where $\left(W_{i j}, \psi_{i j}\right)$ is a foliated chart not necessarily belonging to $\mathcal{U}$.

Let $\pi:(-1,1)^{n} \rightarrow(-1,1)^{q}$ be the natural projection to the last $q$ coordinates. Then the preimage $P_{\lambda}:=\varphi_{\lambda}^{-1}\left(\pi^{-1}(x)\right)$ is called a local leaf. Denote by $Q_{\lambda}$ the space of local leaves. Clearly, $Q_{\lambda} \cong(-1,1)^{q}$, and

$$
U_{\lambda}=\bigcup_{x \in(-1,1)^{q}} \varphi_{\lambda}^{-1}\left(\pi^{-1}(x)\right) .
$$

A foliation $\mathcal{F}$ is said to be oriented if the tangent bundle of $p$-dimensional distribution $T^{\mathcal{F}} M \subset T M$ is oriented, and transversely oriented whenever some transversal to $\mathcal{F}$ distribution of dimension $q=n-p$ is oriented. If the manifold $M$ is Riemannian, then the transverse orientability of $\mathcal{F}$ is equivalent to the transverse orientability of orthogonal distribution $T^{\mathcal{F}^{\perp}} M$.

2.2. Holonomy. We recall the notion of holonomy. Let $l:[0,1] \rightarrow L$ be a closed path in a leaf $L \in \mathcal{F}$. Choose a chain of foliated charts

$$
\mathcal{C}=\left\{U_{0}, \ldots, U_{n-1}, U_{n}=U_{0}\right\}
$$

with the property that there exists a division of the segment

$$
[0,1]: 0=t_{0}<t_{1}<\cdots<t_{n}=1
$$

such that $l\left(\left[t_{i}, t_{i+1}\right]\right) \subset U_{i}$. Then the composition $\bar{g}_{(n-1) n} \circ \ldots \bar{g}_{23} \circ \bar{g}_{12}$ of gluing maps on the space of local leaves, see (2.1) above, is correctly defined in a neighborhood $V_{0} \subset(-1,1)^{q}$ of zero and its germ does not depends on the homotopy class of $[l] \in \pi_{1}(L),[17]$. Denote it by $\Gamma_{l}: V_{0} \rightarrow(-1,1)^{q}$.

Then the correspondence $l \mapsto \Gamma_{l}$ is a homomorphism

$$
\Psi: \pi_{1}(L, x) \rightarrow G_{q}
$$

of the fundamental group $\pi_{1}(L, x)$ at $x$ to the group of germs $G_{q}$ of diffeomorphism at the origin $0 \in \mathbb{R}^{q}$. The homomorphism $\Phi$ is called a holonomy of $L$ and its image, denoted by $H(L)$, is the holonomy group of $L$.

Evidently, they depend on the choice of a point $x \in L$ and a foliated chart $U_{0}$ at $x$. If we choose another point $y \in L$ and another foliated local chart $V_{0}$ at $y$, then $H(L)$ is changed to a subgroup $g \circ H(L) \circ g^{-1}$ of $G_{q}$ for some $g \in G_{q}$. In particular, the isomorphism type of $H(L)$ depends only on the foliation $\mathcal{F}$. 
Notice that if a codimension one foliation is transversely oriented, then the one-sided holonomy

$$
\Psi^{+}: \pi_{1}(L) \rightarrow G_{1}^{+}
$$

of the leaf $L$ is also well defined, where $G_{1}^{+}$denotes the group of germs of one-sided diffeomorphisms at 0 with a domain on the half-intervals $[0, \varepsilon)$.

Nishimori proved the follows theorem describing behavior of a codimension one foliation in a neighborhood of a compact leaf with Abelian holonomy.

Theorem 2.3. [14] Let $\mathcal{F}$ be a transversely oriented $C^{r}$-foliation of codimension one on an oriented $n$-dimensional manifold $M$ and $F_{0}$ be a compact leaf of $\mathcal{F}$. Suppose that $2 \leq r \leq \infty$. Let also $T$ be a tubular neighborhood of $F_{0}$ and $U_{+}$be a union of $F_{0}$ and a connected component $T \backslash F_{0}$. Suppose that $H\left(F_{0}\right)$ is an Abelian group. Then only one of the three cases holds.

1) For any neighborhood $V$ of $F_{0}$ the restricted foliation $\left.\mathcal{F}\right|_{V \cap U_{+}}$has a compact leaf distinct from $F_{0}$.

2) There exists a neighborhood $V$ of $F_{0}$ such that all leaves $\left.\mathcal{F}\right|_{V \cap U_{+}}$ except for $F_{0}$ are dense in $V \cap U_{+}$. In this case, $H\left(F_{0}\right)$ is a free Abelian group of rank $\geq 2$.

3) There exist a neighborhood $V$ of $F_{0}$ and a connected oriented submanifold $N$ of codimension one in $F_{0}$ with the following properties. Denote by $F_{*}$ a compact manifold with boundary obtained by attaching two copies $N_{1}$ and $N_{2}$ of $N$ to $F_{0} \backslash N$ satisfying $\partial F_{*}=N_{1} \cup N_{2}$. Let

$$
f:[0, \varepsilon) \rightarrow[0, \delta)
$$

be a contracting $C^{r}$-diffeomorphism such that $f(0)=0$. Denote by $X_{f}$ a manifold obtained from $F_{*} \times[0, \varepsilon)$ by identifying $(x, t) \in N_{1} \times[0, \varepsilon)$ and $(x, f(t)) \in N_{2} \times[0, \delta)$. After factorization, we obtain the foliation $F_{f}$ on $X_{f}$. Then for some $f$ as above, there is a $C^{r}$-diffeomorphism $h: V \cap U_{+} \rightarrow X_{f}$ which maps each leaf of $\left.\mathcal{F}\right|_{V \cap U_{+}}$onto some leaf of $F_{f}$. The foliation $\left.\mathcal{F}\right|_{V \cap U_{+}}$uniquely defines the homology class $[N] \in H_{n-2}\left(F_{0}, \mathbb{Z}\right)$, and the germ at zero of the map $f$ is unique up to conjugation. In this case, $H\left(F_{0}\right)$ is an infinite cyclic group.

A foliation is said to be a foliation without holonomy if the holonomy of each leaf is trivial, and it is said to be a foliation almost without holonomy if the holonomy of noncompact leaves is trivial. For example, the Reeb foliation $\mathcal{F}_{R}$ is a foliation almost without holonomy on $S^{3}$ since all leaves of $\mathcal{F}_{R}$ except for a single compact leaf being a torus are homeomorphic to $\mathbb{R}^{2}$ and thus have a trivial fundamental group. 
By a block we will means a compact saturated $n$-dimensional submanifold $B$ of a codimension one foliated $n$-dimensional manifold. Recall that a subset of $M$ is saturated with respect to a foliation $\mathcal{F}$ if which is a union of leaves of $\mathcal{F}$. Clearly $\partial B$ is a finite union of compact leaves.

The following statement is a reformulation of results by Novikov [20] and Imanishi [10] obtained for foliations without holonomy and for foliations almost without holonomy, respectively.

Theorem 2.4. Let $L$ be a noncompact leaf of a codimension one foliation $\mathcal{F}$ almost without holonomy on a closed $n$-dimensional manifold $M$. Then one of the following holds:

a) $\mathcal{F}$ is a foliation without holonomy whose all leaves are diffeomorphic to the typical leaf $L$ and dense in $M$. We have the group extension

$$
1 \rightarrow \pi_{1}(L) \rightarrow \pi_{1}(M) \rightarrow \mathbb{Z}^{k} \rightarrow 0,
$$

where $k>0$ and $k=1$ iff the foliation $\mathcal{F}$ is a locally trivial fibration over the circle. Moreover, the universal covering $\widetilde{M}$ has the form

$$
\widetilde{M} \cong \widetilde{L} \times \mathbb{R} .
$$

b) The leaf $L$ belongs to some block $B$ all of whose leaves which contained in the interior $\stackrel{\leftrightarrow}{B}$ are diffeomorphic to the typical leaf $L$ and are either dense in $\stackrel{\circ}{B}$ ( $B$ is called a dense block in this case) or proper in $\stackrel{\circ}{B}(B$ is called a proper block in this case). In both cases we have the group extension

$$
1 \rightarrow \pi_{1}(L) \rightarrow \pi_{1}(B) \rightarrow \mathbb{Z}^{k} \rightarrow 0,
$$

where $k>0$. In this case $k=1$ iff $B$ is proper and the foliation in $\stackrel{\circ}{B}$ is a locally trivial fibration over the circle. Also, the universal covering of each block $\stackrel{\circ}{B}$ has the following form

$$
\widetilde{\circ} \cong \widetilde{L} \times \mathbb{R} \text {. }
$$

2.5. Growth of leaves. A minimal set of the foliation $\mathcal{F}$ is a closed saturated set which has no other closed saturated sets.

The following theorem is a reformulation of [15, Corollary 1.3].

Theorem 2.6. Let $\mathcal{F}$ be a transversely oriented $C^{\infty}$-foliation of a codimension one on a compact manifold $M$. Suppose that every leaf of $\mathcal{F}$ has less than exponential (e.g., polynomial) growth. Then $\mathcal{F}$ does not have any exceptional minimal set. In particular, each minimal set of the foliation $\mathcal{F}$ is either the whole manifold $M$ or a compact leaf.

Here the growth means the volume growth of balls $B_{x}(R) \subset L_{x} \in \mathcal{F}$ as a function of radius $R$. 
A leaf $L$ of a transversely oriented codimension one foliation $\mathcal{F}$ is called resilient if there is a transversal arc $[x, y), x \in L$, and a loop $\sigma$ such that $\Gamma_{\sigma}:[x, y) \rightarrow[x, y)$ is the contraction to $x$, and $L \cap(x, y) \neq \emptyset$.

Theorem 2.7. [8, Proposition 2.1.8] Let $M$ be a compact manifold and $\mathcal{F}$ be a $C^{2}$-foliation of codimension one on $M$. Then a resilient leaf of $\mathcal{F}$ must have exponential growth.

\section{BASIC RESULTS}

Theorem 3.1. Let $M$ be a closed orientable Riemannian 3-manifold $M$ and $\mathcal{F}$ be a codimension one transversely orientable $B$-foliation with respect to the Riemannian metric on $M$. Then

(1) $\mathcal{F}$ is a foliation almost without holonomy;

(2) if a leaf $L^{\prime} \subset \bar{L}$ for some leaf $L \in \mathcal{F}$ then $L^{\prime}$ is flat;

(3) if a leaf $L \in \mathcal{F}$ is non-flat then $L$ is embedded in $M$.

Proof. Let $L \in \mathcal{F}$ be a noncompact leaf. Suppose that there exists another leaf $L^{\prime} \in \bar{L}$ and a point $x \in L^{\prime}$ such that Gauss curvature $K_{L^{\prime}}(x) \neq 0$. Notice that the induced Riemannian metric smoothly depends on leaves and the leaf curvature function $K_{L_{y}}(y), y \in M$ is continuous on $M$. Then $K_{L_{y}}(y)$ is bounded, since $M$ is compact. Suppose that $\left|K_{L_{y}}(y)\right|<C$ at any point $y \in M$.

There exists a foliated compact normal neighborhood $V(x)$ of $x$ and a homeomorphism $f: I \times D^{2}(r) \rightarrow V(x)$, where $I=[0,1], D^{2}(r)$ is the standard Euclidean disk of radius $r$, and $f(I \times 0)$ is a regular parametrization of an arbitrary small path $\gamma$ passing through $x=f(1 / 2,0)$ and orthogonal to $\mathcal{F}$.

Denote $D_{t}^{2}(r)=t \times D^{2}(r)$ and let $L_{t}$ be the leaf of $\mathcal{F}$ containing $f(t, 0)$, $t \in I$. The normality of the neighborhood $V(x)$ means that the disks $f\left(D_{t}^{2}(r)\right) \subset L_{t} \in \mathcal{F}$ are normal neighborhoods of points $f(t, 0)$ in the leaves $L_{t}, t \in I$, which are defined by the tangent to $\mathcal{F}$ exponential map $\left.\exp _{\mathcal{F}}\right|_{\gamma}: T \mathcal{F} \rightarrow M$ as follows: $\exp _{\mathcal{F}}$ maps a tangent to $\mathcal{F}$ vector $v \in T_{x} \mathcal{F}$ with the origin at $x \in M$ to $\alpha_{v}(1)$, where $\alpha_{v}$ is a geodesic on the leaf $L_{x}$ defined by the initial conditions $\alpha_{v}(0)=x$ and $\alpha_{v}^{\prime}(0)=v$. Choosing $\gamma$ and $r$ we can make $V(x)$ so small that $K_{L_{y}}(y) \neq 0$ for each $y \in V(x)$.

Now we can take $r$ small enough so that $\operatorname{Area}_{L_{t}}\left(D_{t}^{2}(r)\right), t \in I$, are not less than the area of an embedded disc of radius $r$ in a simply connected manifold of constant curvature $C$. It follows from the well known formula for the area of a small ball of radius $r$ at a point $p$ of a Riemannian surface 
$M$ with a function of curvature $K(x), x \in M$, that

$$
\operatorname{Area}_{M}\left(D^{2}(r)\right)=\operatorname{Area}_{E^{2}}\left(D^{2}(r)\right)\left(1-\frac{K(p)}{12} r^{2}+o\left(r^{2}\right)\right) .
$$

Thus there is a constant const ${ }_{1}$ such that $\operatorname{Area}_{L_{t}}\left(D_{t}^{2}(r)\right)>$ const $_{1}>0$, $t \in I$. Since $L^{\prime} \in \bar{L}$ we can find a countable number of disks $D_{t_{i}}^{2}(r) \subset L$, $t_{i} \in I, i=1, \ldots,+\infty$. It means that the total absolute curvature

$$
\int_{L}|K| d \mu>\min _{x \in V_{x}}|K|_{L_{x}}(x) \sum_{i=1}^{\infty} \operatorname{Area}_{L}\left(D_{t_{i}}^{2}(r)\right)=\infty .
$$

But it contradicts to the assumption and we conclude that $L^{\prime}$ is flat. In particular, this implies that limit compact leaves are flat tori. According with Theorem 2.3 the leaf $L$ is either locally dense or embedded. Since a locally dense leaf contained in the boundary of another leaf it has to be flat, which proves (2). It also follows that a not flat leaf has to be embedded into $M$, and this proves (3).

To prove (1) suppose that $L$ has nontrivial holonomy. Since $\mathcal{F}$ is transversely oriented and a nontrivial periodic diffeomorphism of an interval should be an involution relative to the origin, the holonomy must be infinite. Let $\gamma$ be a closed path in $L$ representing a nontrivial holonomy element.

Case 1. Suppose that the one-sided holonomy $\Psi^{+}([\gamma])$ is nontrivial and the holonomy map $\Gamma_{\gamma}:[0, \varepsilon) \rightarrow\left[0, \varepsilon^{\prime}\right)$ does not have any fixed points in $[0, \varepsilon)$ for some $\varepsilon$.

In this case, $\Psi^{+}( \pm[\gamma])$ is represented by a contracting map. If $L$ is locally dense, then $L$ must be a resilient leaf, which is impossible by Theorem 2.7. Recall that by Theorem 1.4 the leaves of $\mathcal{F}$ have polynomial grows. Otherwise, another noncompact leaf $P$ would wind around $L$ and by Theorem 2.6 one could find a compact leaf $K \subset \bar{L} \bigcap \bar{P}$. It simply follows from part 3) of Theorem 2.3 that the leaf $P$ must have infinitely many ends, which is impossible as leaves of $\mathcal{F}$ have a finite topology (see $\S 1$ ).

Case 2. The half-interval $[0, \varepsilon)$ contains a sequence of fixed points $\left\{F_{i}\right\}$ of the holonomy map $\Gamma_{\gamma}:[0, \varepsilon) \rightarrow\left[0, \varepsilon^{\prime}\right)$ which converges to zero.

Since $\left\{F_{i}\right\} \bigcap\left[0, F_{k}\right]$ is closed in $[0, \varepsilon)$, where $F_{k} \in\left\{F_{i}\right\} \bigcap[0, \varepsilon)$, it follows that either the holonomy of $L$ is trivial and this proves the claim or we have to find a half-interval $[a, \delta) \subset[0, \varepsilon)$ on which $\Gamma_{\gamma^{\prime}}$ is a contracting map. The leaf $L^{\prime}$ corresponding to the point $a \in[0, \varepsilon)$ has a contracting holonomy on $\gamma^{\prime} \subset L^{\prime}$, where $\gamma^{\prime}$ is a closed path corresponding to the fixed point $a$ of the map $\Gamma_{\gamma}$. Since the set of compact leaves is closed (see [17]) and $M$ is a normal topological space, we can choose $\varepsilon$ small enough for the leaf $L^{\prime}$ to be noncompact. Thus we are under assumptions of Case 1 which proves Theorem 3.1. 
3.2. Proof of Main Theorem. (1) Statement (a) follows from Theorem 1.5 and (b) is a consequence of Theorem 3.1.

(c) Suppose that all leaves of the foliation $\mathcal{F}$ are compact. Then from orientibility of both $\mathcal{F}$ and $M$ we obtain that each leaf is orientable and has trivial holonomy. Hence by Reeb stability Theorem $\mathcal{F}$ is a fiber bundle over the circle. Since the leaves according to the condition of the Main Theorem have nonpositive curvature, we obtain that each fiber must be homeomorphic to a surface of genus $g \geq 1$ due to Gauss-Bonnet Theorem. This proves substatement (i).

Suppose that $\mathcal{F}$ does not contain compact leaves. By Theorem $3.1 \mathcal{F}$ is a foliation without holonomy. Hence it follows from Theorem 2.4 that all leaves are dense. In this case by Theorem 3.1 each leaf of $\mathcal{F}$ is flat and $M$ must be a torus bundle over the circle (see [2, Paragraph 4]). This proves substatement (ii).

To establish (iii) assume that $\mathcal{F}$ contains both compact and noncompact leaves. It can be shown that proper blocks described in the Theorem 2.4 satisfy the condition described in (iii). Indeed, the boundary of a proper block $B$ is a family of limit compact leaves which must be a family of flat tori by Theorem 3.1. The foliation $\mathcal{F}$ in a collar of the torus $T^{2} \subset \partial B$ is described in the part 3) of Theorem 2.3. It allows us to construct a family of tori transversal to $\mathcal{F}$ and dividing $B$ into two parts:

- one of which is a disjoint union of finitely many copies of $T^{2} \times I$,

- and another one is homeomorphic to $B$ and has an induced from $\stackrel{\circ}{B}$ structure of a fibre bundle over the circle with the fiber being a compact surface with boundary.

Hence we get from Theorem 3.1 that a dense block (see Theorem 2.4 for definition) consists of flat leaves and as proved in [2] it must be homeomorphic to one of the following manifolds:

$$
T^{2} \times I \quad D^{2} \times S^{1} \quad \mathcal{K},
$$

where $\mathcal{K}$ is a nontrivial $I$-bundle over Klein bottle. But the fundamental group of a dense block $B$ has a form of the extension (2.3) with $k>1$, whence we immediately conclude that the only possibility is $B \cong T^{2} \times I$. Recall also that the set of compact leaves is closed in $M$ and therefore we can divide $M$ into finitely many blocks which either are such described above or they are arbitrary thin blocks containing infinitely many compact leaves. It is easy to see that each thin block is homeomorphic to the direct product of a compact leaf with a closed interval $K \times I$ (see [2, Paragraph 4]).

The structure of fundamental group $\pi_{1}(M)$ immediately follows from Theorem 1.6 and [6, Th.6.2.11]. This completes (iii). 
(2) To prove (a) we will construct a $B_{\leq 0}$-foliation in each block $A_{j}$ and then connect those blocks preserving Riemannian metric on the leaves by gluing of $\left(T^{2} \times I\right)$-blocks via the corresponding diffeomorphisms to get a manifold diffeomorphic to $M$. Since each $A_{j}$ is homeomorphic to some $S$ bundle $E$ over the circle, the idea is to construct the Riemannian metric on $E$ which is locally Euclidean on collars of boundary tori of $E$ so that the fibers of $E$ will have nonpositive curvature and will be orthogonal to the flat boundary tori in the collars. After that we will construct $B_{\leq 0^{-}}$ foliation on $E$, making the ends of the fibers endless euclidean cylinders winding around boundary tori. Note that that we can choose two opposite direction of the winding to define agreed orientation on the boundary tori of adjacent blocks.

Firstly suppose that $S$ is a compact surface with a boundary $\partial S$ consisting of $n$ disjoin circles and $\chi(S)<0$. Let $\operatorname{Diff}_{+}(S)$ be a group of orientation preserving smooth diffeomorphisms of $S$ with the $C^{\infty}$-topology of uniform convergence on compact sets of all differentials. Assume that some block $A_{j}$ from Main Theorem is homeomorphic to a locally trivial $S$-bundle $E$ over the circle $B$. Then there exists $f \in \operatorname{Diff}_{+}(S)$ such that

$$
E=S \times I / s \times 0 \sim f(s) \times 1, \quad B=I /\{0,1\} \cong S^{1} .
$$

The diffeomorphism $f$ induces a permutation $\sigma: A \rightarrow A$ of the finite collection $A$ of connected components of $\partial S$. Each orbit of this permutation corresponds to one boundary component of $\partial E$, which is diffeomorphic to the 2-torus $T^{2}$ since $f$ preserves orientation of $S$.

The following principal $\operatorname{Diff}_{0}(S)$-fibre bundle holds, [4]:

$$
\operatorname{Diff}_{0}(S) \rightarrow \mathcal{M e t}_{-1}(S) \rightarrow \mathcal{T}(S),
$$

where $\operatorname{Diff}_{0}(S) \subset \operatorname{Diff}_{+}(S)$ is the path component of the identity map in $\operatorname{Diff}_{+}(S)$, $\mathcal{M e t}_{-1}(S)$ is the space of hyperbolic structures (with geodesic boundaries) on $S$, and $\mathcal{T}(S)$ is the Teichmüller space of smooth conformal structures on $S$. Recall that a space of smooth conformal structures on $S$ coincides with $\mathcal{M e t}_{-1}(S)$ if $\chi(S)<0$, see [18]. It follows from the uniformization Theorem for compact Riemann surfaces with boundary claiming that every such surface (except for the disk and annulus) admits a unique conformal hyperbolic metric with geodesic boundary. In this case $\operatorname{Diff}_{0}(S)$ and $\mathcal{T}(S)$ are contractible and so is the Frechet manifold $\mathcal{M e t}_{-1}(S)$, see [4]. Moreover, $\mathcal{T}(S)$ is homeomorphic to $\mathbb{R}^{6 g-6+3 n}$ with the Fenchel-Nilsen coordinates, see [18] and [12], where the last $n$ coordinates correspond to the lengths of the boundary circles of $\partial S$. Suppose that the lengths of boundary circles are fixed and equal the same constant $l$. Obviously, in this case the corresponding Teichmüller space $\mathcal{T}_{l}(S)$ is homeomorphic to $\mathbb{R}^{6 g-6+2 n}$ and the restriction $\mathcal{M e t}_{-1}^{l}(S)$ of $\mathcal{M e t}_{-1}(S)$ to $\mathcal{T}_{l}(S)$ is contractible also. In 
particular we can join the points $g_{0}$ and $g_{1}=f^{*} g_{0}$ in $\mathcal{M e t}_{-1}^{l}(S)$ by some smooth path $g_{t}$ in $\mathcal{M e t}_{-1}^{l}(S)$ to obtain a Riemannian metric on $E$ having the following form in the corresponding local coordinate system:

$$
d s^{2}=d t^{2}+g_{t} .
$$

Notice that the metric of this kind depends on a choose of a horizontal (i.e. orthogonal to the fibers of $p: E \rightarrow B$ ) one-dimensional distribution, [1]. For any such metric the projection $p: E \rightarrow B$ becomes a Riemannian submersion with hyperbolic fibers $\left(S_{t}, g_{t}\right)$ having geodesic boundaries of length $l$.

Consider the boundary torus $T \subset \partial E$, and let

$$
V \cong[0, \delta) \times C_{1} \times C_{2}
$$

be a collar of $T$, where $C_{i}, i=1,2$, are circles such that $[0, \delta) \times C_{1} \times s$ belongs to the fiber for each $s \in C_{2}$. Then

$$
V \cap S_{t} \cong \bigsqcup_{i=1}^{k}\left([0, \delta) \times C_{1} \times s_{i}\right),
$$

where $k$ is the number of elements of the corresponding orbit of $\sigma$. We can assume that $f^{k}$ is the identity diffeomorphism for the corresponding $k$ in the collar of each boundary component of $\partial S$.

Let us choose a half-geodesic coordinate system in some neighborhood $U_{s}$ of the boundary of each level set $[0, \delta) \times C_{1} \times s$. We obtain the hyperbolic metric on $U_{s}$ having the same form for arbitrary $s \in C_{2}$, [21]:

$$
d s^{2}=d r^{2}+\operatorname{ch}^{2}(r) d \phi^{2},
$$

where $r \in[0, R], \phi \in[0, l]$. Assume that the circle $C=0 \times 0 \times C_{2}$ consists of origins of the coordinate systems in $U_{s}$. Notice that the submersion $p: E \rightarrow B$ restricted on $C$ is a covering map and we can assume that the parameter $s$ is locally the same as the parameter $t$ on base $B$. Moreover, if $t \in[0,1]$ parameterizes the circle $B$, then $s \in[0, k]$ parameterizes the circle $\mathrm{C}_{2}$.

Consider the collar $W=\bigcup_{s} U_{s}$ of $T$ and define the flow $\left\{\Phi_{t}\right\}, t \in \mathbb{R}$, on $W$ by the equality of half-geodesic coordinates. Here the parameter $t$ corresponds to the parameter on the universal covering $\mathbb{R}$ of $B$. It means that if $x \in U_{s}$ has half-geodesic coordinates $(r, \phi)$, then $\Phi_{t}(x) \in U_{(s+t) \bmod k}$ and has the same half-geodesic coordinates. The flow $\left\{\Phi_{t}\right\}$ is smooth since the Riemannian metric $g_{t}$ is changed smoothly with $t$. Evidently, trajectories of $\left\{\Phi_{t}\right\}$ define a transversal distribution on $W$ homotopic to the horizontal distribution. We can extend that homotopy remaining identical outside of $V$ to obtain a smooth transversal distribution on the whole $E$. 
Let us change the Riemannian metric (3.1) so that the resulting distribution becomes horizontal (i.e. orthogonal to fibers). Clearly, the flow $\left\{\Phi_{t}\right\}$ preserves the new Riemannian metric on $W$ being the metric of a direct product

$$
d s^{2}=d r^{2}+\operatorname{ch}^{2}(r) d \phi^{2}+d t^{2}
$$

in the new coordinate system $(r, \phi, t)$. Let us change the metric $d s^{2}$ replacing the function $\operatorname{ch}^{2}(r)$ by a smooth convex function such that (see Figure 3.1):

$$
h(r)= \begin{cases}\operatorname{ch}(c), & 0 \leq r \leq r_{1} \\ \operatorname{convex}, & r_{1} \leq r \leq r_{2} \\ \operatorname{ch}(r), & r_{2} \leq r \leq R\end{cases}
$$

where $r_{1}<c<r_{2}$.

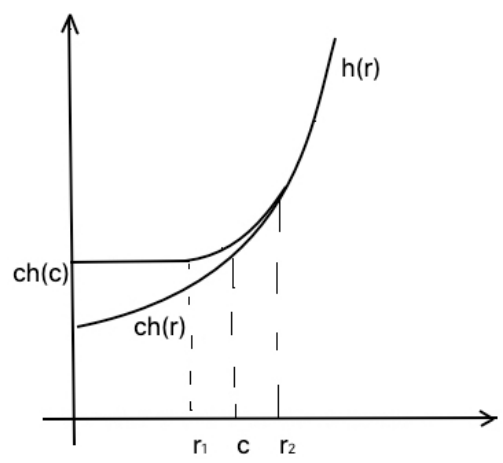

FigURE 3.1. Graph $h(r)$

Since $h$ is convex, the curvature of the metric $d s^{2}=d r^{2}+h^{2}(r) d \phi^{2}$ is nonpositive and it is flat for $0 \leq r \leq r_{1}$ as well as the curvature of the metric $d s^{2}=d r^{2}+h^{2}(r) d \phi^{2}+d t^{2}$.

The leaves of the fiber bundle $p: E \rightarrow B$ are level hypersurfaces $t=\mathrm{const}$ in $W(r, \phi, t)$ wiht respect to the new Riemannian metric. Let us do the turbulisation of this fibration around of $T$ to obtain a foliation on $W$ with a compact leaf $T$. For this define the foliation by the kernel of the 1-form

$$
\omega(r)=(1-f(r)) d r \pm f(r) d t,
$$

where $f:[0,1] \rightarrow[0,1]$ is an arbitrary smooth function such that

$$
f(r)= \begin{cases}0, & 0 \leq r \leq r_{4} \\ \text { increasing, } & r_{4} \leq r<r_{3} \\ 1, & r_{3} \leq r \leq R,\end{cases}
$$


and $r_{4}<r_{3}<r_{1}$.

The sign in the definition of $\omega$ is chosen depending on the direction of the winding.

The kernel $\operatorname{ker}(\omega)$ defines a trivial foliation $\{r \times T\}$ in the neighborhood $0 \leq r \leq r_{4}$ of the compact leaf $T$ and the foliation by euclidean cylinders in the domain $r_{4}<r \leq r_{1}$. One can see that leaves of $\operatorname{ker}(\omega)$ are flat in the domain $0 \leq r \leq r_{1}$. Repeating the arguments, we will obtain a foliation of nonpositive curvature on $E$ such that the boundary of $E$ consists of compact leaves. Notice that curvature of each noncompact leaf has compact support. This proves the case $(2)_{(\text {a) }}$ when $\chi(S)<0$.

Notice that the case when $S$ is a 2-disk (i.e. $\chi(S)>0$ ) is not possible. Indeed, then the block should be homeomorphic to a Reeb component (see [20]) and the homomorphism $i_{*}: \pi_{1}\left(T^{2}\right) \rightarrow \pi_{1}(B)$ induced by the inclusion $i: T^{2} \rightarrow B$ must have a nontrivial kernel which contradicts to Theorem 1.6.

Also if $S$ is annulus (i.e. $\chi(S)=0$ ), then $B$ is homeomorphic to $T^{2} \times I$ or $\mathcal{K}$ (see above), which admit a flat proper foliation as was shown in [2].

It remains to glue two blocks (or single block) by a family of flat tori $T^{2} \times I$. Recall that the space of flat Riemannian metrics $\operatorname{Met}_{0}\left(T^{2}\right)$ is homeomorphic to

$$
\mathbb{R}_{+} \times \operatorname{Met}_{0}^{1}\left(T^{2}\right),
$$

where $\mathcal{M e t}_{0}^{1}\left(T^{2}\right)$ is the space of all flat metrics on $T^{2}$ with unit area. At the same time there is a fiber bundle

$$
\operatorname{Diff}_{0}\left(T^{2}\right) / T^{2} \rightarrow \mathcal{M e t}_{0}^{1}\left(T^{2}\right) \rightarrow \mathcal{T}\left(T^{2}\right),
$$

where $\mathcal{T}\left(T^{2}\right)$ is the Teichmüller space of $\mathcal{T}\left(T^{2}\right)$ which is homeomorphic to Lobachevski plane $H^{2}$, and the factor-group $\operatorname{Diff}_{0}\left(T^{2}\right) / T^{2}$ is contractible, [5]. Here we identify $T^{2}$ with the subgroup of torus motions in $\operatorname{Diff}_{0}\left(T^{2}\right)$. We conclude that the space $\mathcal{M e t}_{0}\left(T^{2}\right)$ is contractible. Therefore we can join any two flat metrics $g_{0}$ and $g_{1}$ on $T^{2}$ by a smooth path $g_{t}$ in $\mathcal{M e t}_{0}\left(T^{2}\right)$ which gives a family of tori $I \times T^{2}$ with the metric $d t^{2}+g_{t}$. Pasting the cylinders $I \times T^{2}$ with corresponding Riemannian metrics between blocks we obtain the proof of $(2)_{(a)}$.

In particular, we also obtain a flat foliation on any torus bundle which partially proves $(2)_{(b)}$.

The same arguments applied to the spaces $\mathcal{M e t}_{-1}\left(M_{g}^{2}\right)$, where $M_{g}^{2}$ is a closed orientable surface of genus $g>1$, give us a foliation by fibers of constant curvature -1 on a mapping torus

$$
M_{f}=I \times M_{g}^{2} /_{(0, x) \sim(1, f(x))} .
$$


Recall that $\mathcal{M e t}_{-1}\left(M_{g}^{2}\right)$ is contractible, [5]. The Riemannian metric $d t^{2}+g_{t}$ on $M_{f}$ is defined by the family metrics $g_{t}, t \in[0,1]$ of constant curvature on $t \times S$ connecting the metrics $g_{0}$ and $g_{1}=f^{*} g_{0}$. It finishes the proof of $(2)_{(b)}$ and Main Theorem.

Corollary 3.3. Not all hyperbolic forms admit B-foliation.

Proof. Indeed, it is well known that the fundamental group of a hyperbolic form does not contain $\mathbb{Z} \oplus \mathbb{Z}$ (see [18]). Thus the realizable case is $(1)_{(\mathrm{c})(\mathrm{i})}$ only.

Remark 3.4. Noice that such hyperbolic forms exist, [11], but there are many hyperbolic forms with a finite first homology group, [19], and which therefore cannot be fibered over the circle. Note also that not all spherical forms admit nonnegative curvature foliations, see [2].

Remark 3.5. All closed oriented 3-manifolds $M$ admit $B$-foliation. This immediately follows from open book decomposition theorem, which allows us to decompose $M$ into fibered pieces including solid tori with Reeb foliations, which admit nonnegative curvature, see [2].

The author is grateful to Misha Kapovich for consultations on Teichmüller spaces and to Sergiy Maksymenko for useful remarks.

\section{REFERENCES}

[1] Arthur L. Besse. Einstein manifolds, volume 10 of Ergebnisse der Mathematik und ihrer Grenzgebiete (3) [Results in Mathematics and Related Areas (3)]. Springer-Verlag, Berlin, 1987.

[2] D. V. Bolotov. Foliation of nonnegative curvature on closed 3-manifolds. Mat. Sb., 200(3):3-16, 2009.

[3] Stefan Cohn-Vossen. Kürzeste Wege und Totalkrümmung auf Flächen. Compositio Math., 2:69-133, 1935.

[4] C. J. Earle, A. Schatz. Teichmüller theory for surfaces with boundary. J. Differential Geometry, 4:169-185, 1970.

[5] Clifford J. Earle, James Eells. A fibre bundle description of Teichmüller theory. J. Differential Geometry, 3:19-43, 1969.

[6] Ross Geoghegan. Topological methods in group theory, volume 243 of Graduate Texts in Mathematics. Springer, New York, 2008.

[7] Philip Hartman. Geodesic parallel coordinates in the large. Amer. J. Math., 86:705$727,1964$.

[8] Gilbert Hector, Ulrich Hirsch. Introduction to the geometry of foliations. Part B. Aspects of Mathematics, E3. Friedr. Vieweg \& Sohn, Braunschweig, second edition, 1987. Foliations of codimension one.

[9] Alfred Huber. On subharmonic functions and differential geometry in the large. Comment. Math. Helv., 32:13-72, 1957.

[10] Hideki Imanishi. Structure of codimension one foliations which are almost without holonomy. J. Math. Kyoto Univ., 16(1):93-99, 1976. 
[11] Troels Jørgensen. Compact 3-manifolds of constant negative curvature fibering over the circle. Ann. of Math. (2), 106(1):61-72, 1977.

[12] M. Kapovich. Introduction to the Teichmuller theory. Preprint, 1997.

[13] Peter Li. Complete surfaces of at most quadratic area growth. Comment. Math. Helv., 72(1):67-71, 1997.

[14] Toshiyuki Nishimori. Compact leaves with abelian holonomy. Tôhoku Math. J. (2), 27(2):259-272, 1975.

[15] J. F. Plante. On the existence of exceptional minimal sets in foliations of codimension one. J. Differential Equations, 15:178-194, 1974.

[16] Garrett Stuck. Un analogue feuilleté du théorème de Cartan-Hadamard. C. R. Acad. Sci. Paris Sér. I Math., 313(8):519-522, 1991.

[17] Itiro Tamura. Topology of foliations: an introduction, volume 97 of Translations of Mathematical Monographs. American Mathematical Society, Providence, RI, 1992. Translated from the 1976 Japanese edition and with an afterword by Kiki Hudson, With a foreword by Takashi Tsuboi.

[18] William P. Thurston. Three-dimensional geometry and topology. Vol. 1, volume 35 of Princeton Mathematical Series. Princeton University Press, Princeton, NJ, 1997. Edited by Silvio Levy.

[19] А. Ю. Веснин, Т. А. Козловская. Разветвленные циклические накрытия линзовых пространств. Сибирский математический журнал, 52(3):542- 554, 2011.

[20] С. П. Новиков. Топология слоений. Труды Московского математического общества, 14:249-278, 1965.

[21] А. В. Погорелов. Дифференциальная геометрия. М: Наука, 1974.

Received: October 18, 2018, accepted: December 3, 2018.

Dmitry V. Bolotov

Department of Differential Equations and Geometry

B. Verkin Institute for Low Temperature Physics and Engineering, National ACADEMy of Sciences of Ukraine

47 Nauky Ave., Kharkiv, 61103, Ukraine

Email: bolotov@ilt.kharkov.ua 